\title{
Daily Orders Part - I a Simple Web based Information System
}

\author{
Nibeesh K \\ Senior Technical Assistant 'B' \\ Combat Vehicles Research and \\ Development Establishment, \\ DRDO \\ Chennai -54, Tamil Nadu, India
}

\begin{abstract}
CVRDE Employees were finding it difficult to obtain Daily Orders Part I information by searching the relevant matter through bounded hard copies.

Similarly, Establishment found difficulties in generating more than 100 copies of each Daily Orders Part I and distributes the same to each section within the stipulated time [10].

Hence, a web based new system is developed with search facility. This system eliminates the difficulties of accessibility and scalability in the existing business process.

\section{General Terms}

Daily Orders Part I: It is a document which is prepared, published and circulated by the Establishment regarding administrative news, orders, promotions, committees, duties, transfers, appointments, forums, leave etc.
\end{abstract}

\section{Keywords}

Daily Orders Part I, Oracle, PLSQL, BLOB, CLOB, ASP.NET.

\section{INTRODUCTION}

All Organization/Establishment have Information systems. The aim of the information system is to make reach up-to-date information's within the establishment.

The successful implementation of computer information system requires proper knowledge of the existing system, user level of intelligence, requirement of the user and the management. In the system analysis phase, we under stood that, task holders are not mentally inclined to the changes of their routine works[14] and the user need the information should available over campus network with various combination of search, such as date, keyword must exist (optional) and at least some keywords exist (optional).

From the above study it is proposed to develop a web based application [9][13]. Web development is more complicated than traditional software development due to numerous factors [9][17].

In the new web based application there are mainly two interfaces, one is to view[24] and search the Daily Orders Part I and the other is to add information regarding present publishing details and files.

There are two levels of login:

1. Task holder: Using task holders' login he/she can attach scanned pdf and word file with particulars of Daily Orders Part I number and release after verification.
2. Administrator: Administrator has the rights to add/remove new task holder. He/she can also delete, modify the information in the current releases .This helps to correct any mistake happened in the current release.

\section{ACHIEVEMENT}

After the implementation of new web based information system, establishment achieved the following:

1. Consumption of paper has been brought down [11] to $2 \%$.

2. Man hours saved for circulation.

3. Man hours saved in keeping and searching the files.

4. Ink consumption reduced to $1 \%$.

5. All time availability over the campus network.

Prior to this every month, around five reams of paper were used for circulation. Now only signed master copy is maintained. Employees find the search facility as the most useful tool for obtaining the information relevant to old Daily Orders Part I.

\section{SOFTWARE/LIBRARIES USED}

To develop the new system in web based three tire architecture [12], HTML, ASP.NET, Oracle and JavaScript were used. Microsoft's web technologies with Oracle are selected because of the following reasons:

- Other web applications in this Establishment are based on this technology [12].

- $\quad$ Easy in development, testing and debugging [21].

- Fastness.

- Many help documents with sample codes are available in the Internet.

\subsection{IIS 7.0}

Internet Information Services (IIS)-Internet Information Server is a web server application by Microsoft with increased support for the .NET framework to use with Microsoft Windows. IIS is the third most popular server in the world. Using the hierarchical configuration system manager we can deploy as web site easily. IIS 7.0 includes a new modular design, that reduces surface attack as well as it increases performance.

\subsection{VISUAL STUDIO 2008}

Microsoft Visual Studio 2008 [1][2] is an Integrated Development Environment (IDE) which provides functionality for building .NET applications by using .NET 
languages. It provides a better debugging facility to the developers. We can use Visual Studio 2008 to target multiple .NET Framework versions by allowing building and maintaining applications for .NET 2.0 and .NET 3.0 in addition to its native and default support for .NET 3.5.

\subsection{ASP.NET 3.5, C\# 3.0}

ASP.NET is a web application framework developed by Microsoft to build dynamic Websites, web applications and web services. ASP.NET is built on the Common Language Runtime [20] that allows programmers to use objects written in different .NET languages. .NET framework aims for performance benefits over other script based technologies (including Classic ASP) by compiling the server side code to one or more DLL files on the web server.

$\mathrm{C \#}$ is one of the widely used .NET language .It is a type-safe, object-oriented languages .It enables developers to build secure and robust applications. It adopted many syntax from $\mathrm{C}++$. C\# have powerful features such as nullable value types, enumerations, delegates, lambda expressions and direct memory access. It has many innovative language constructs such as delegates, Properties, Attributes, Inline XML documentation comments , Language-Integrated Query (LINQ),etc.

\subsection{Oracle 11g}

The Oracle database is an object relational database management system (ORDBMS) by Oracle Corporation. Oracle is a major platform independent database management system .Oracle have lot of added features with the ACID[5] properties which is important in insuring the integrity of data.

Oracle Database 11g makes database infrastructure far more efficient, resilient, and manageable by the added new features such as Database Replay, SQL Access Advisor, PL/SQLEfficient Coding and Compression.

To store large unstructured Data and Semi-structured Data[8] ,oracle provides two data types, BLOB and CLOB .These are commonly called as Large Objects(LOBs) and can hold up to a maximum size ranging from 8 terabytes to 128 terabytes ,depending on how your database is configured. Storing data in LOBs enables you to access and manipulate the data efficiently in your application.

\subsubsection{BLOB}

Stores any kind of data in binary format. Typically used for multimedia data ,such as images, audio, and video.

\subsubsection{CLOB}

Stores string data in the database character set format. Used for large strings or documents that uses the database character set exclusively.

\section{5 jQuery 1.7}

jQuery [6] is a new kind of Cross browser JavaScript library. Using jQuery we can develop fast and concise JavaScript codes to HTML document traversing, event handling [7], animating, widgets[22], and Ajax interactions. It is free, open source software Dual-licensed under the MIT license and the GNU General Public License.

\subsection{Office 2007 Primary Interop Assemblies}

The Office 2007 Primary Interop Assemblies helps to use the features of Microsoft Office application from an Office project. The PIA enables managed code to interact with Microsoft Office application's COM-based object model.

\section{FLOW OF SYSTEM}

The flow of the system is as shown in Fig. 1.

When task holder attaches a file, it is saving in database as well as in the folder. When the file is retrieved it is fetched from database only.

1. Saving file in the folder using "Fileupload" control.

2. Saving BLOB for pdf, word file and CLOB for word file, other information and with heading.

3. Selecting record based on the keywords using AND and OR Search.

4. Administrator can correct any mistake in the information.

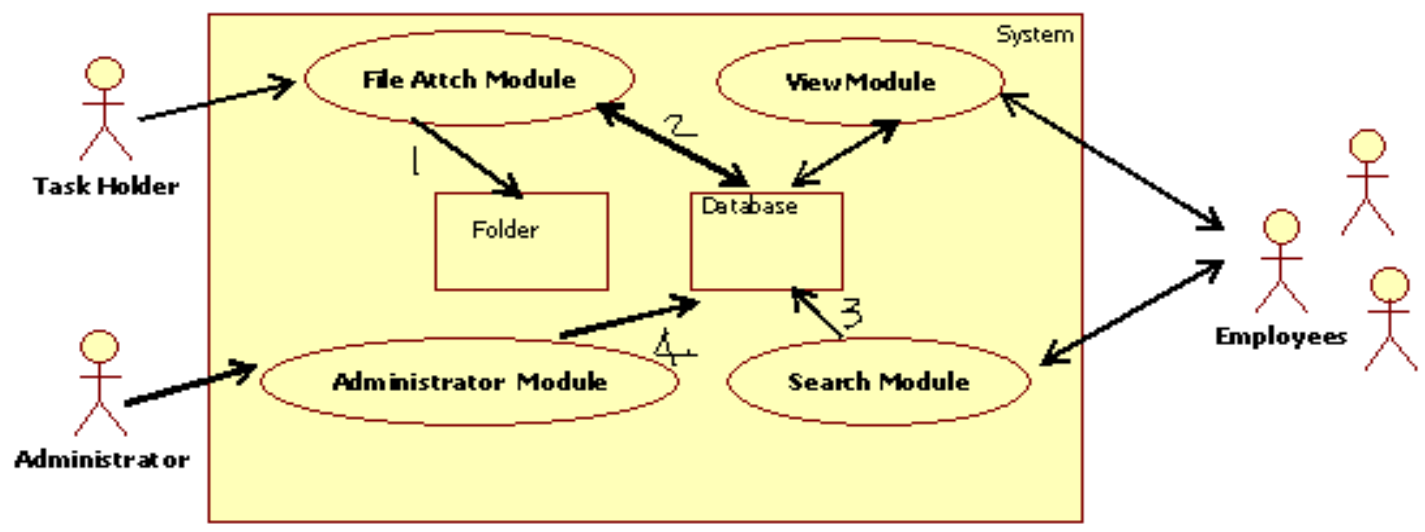


Fig 1: System Flow.

\section{TABLE STRUCTURE}

DO_PART is the main table used for this system implementation. This table contains DO_NUMBER, DATE, STATUS, TYPE with the two BLOB to store pdf, word file and two CLOB to store word file contents and heading details.

For user login and transaction details, the tables, procedure, and code by existing login system reused.

\section{CONFIGURATION}

Every application requires some sort of configuration .The configuration required for this application is explained below.

\subsection{Web.config}

Web.config[18] is web application configuration file used by ASP.NET web application. It contains many configuration details for the application.

\subsubsection{HTTP RUNTIME[19]}

In the web.config under <system.web> should add

$<$ httpRuntime executionTimeout="400"

maxRequestLength="51200"/> .

This will increase the processing time of the web page and the maximum size of the file that we can upload as explained below.

a) executionTimeout: Used to mention the maximum seconds a request is allowed to execute..

b) maxRequestLength: Used to mention the maximum file upload size by the application. The size specified is in kilobytes. The default is $4096 \mathrm{~KB}$ (4 MB).

\subsubsection{CONNECTION STRING}

In web.config under <configuration>, <connectionStrings> add two names one for database connection and other for the folder location(with name DO_PATH) to server local drive where the uploaded files stores.

\subsection{Folder}

In $\mathrm{C}$ drive of the system folder with name DO_PART is created that will store all the files which are uploaded including word and pdf with unique Daily Orders Part I no. and file extensions. To access this folder from the web application , access permission to be given to IIS. To give permission Right click on mouse->properties->security>add$>$ advanced->find now then add

\section{IIS_IUSERS( "Your machine name"'IIS_IUSER) \\ 2. IUSER}

Select each user and give full control.

\subsection{Office 2007 Dcom}

\subsubsection{Install Microsoft Office Word 2007.}

6.3.2 Down load Office 2007 Interop Assemblies redistributable package.

\subsubsection{Configure word DCom described as bellow}

1) Go to run in the start menu.

2) Type "dcomenfg" then press enter key

3) From the displaying window go to "component services->computers->My computers-DCOM Config then a warning window will be displayed come, click ok.

Now from the displayed components, search for Application Name: "Microsoft Office Word 972003 Document" or Application ID: \{000209060000-0000-C000-000000000046\}. Select the component and go for properties ->security. There are three sections:

a) Launch and Activation Permission.

b) Access Permission.

c) Configuration Permission

Click edit button->security->add->Advanced->Find now from the list select

- Administrator("Your machine name"।

Administrator)

- Administrators("Your machine name")

Administrators)

- INTERACTIVE

- IIS_IUSERS( "Your machine name”IIIS_IUSER)

- IUSER

- SYSTEM

- NETWORK

4) Select each user and select allow radio button. Then press OK.

5) The above configuration should be repeated for each sections $\mathrm{a}, \mathrm{b}$ and $\mathrm{c}$.

6) From the properties go to "Identity" select "This user" give machine administrator name and password press Apply then save.

7) Restart the computer.

\section{CODE SNIPS}

\subsection{File upload}

In ASP.NET we can upload file using the "Fileupload" control. There are two file controllers used, fuPdf for pdf and fuWord for word. The files are saved to the folder as

\footnotetext{
pdfExtention $=$ fuPdf.FileName,SubString(fuPdf.FileName LastIndexOf(“.”));

fu.Pdf.SaveAs(DO_PATH+do_nimber + fileExtention);
}

DO_PATH is the path to local folder. This value is from the connection variable DO_PATH of web.config

\subsection{Extracting Word Data}

\# region "Reading word data..."

Microsoft.Office.Interop.Word.ApplicationClass wordApp = new Microsoft.Office.Interop.Word.ApplicationClass(); object file DO_PATH + do_nimber + word_Extesion; object nullobj = System.Reflection.Missing.Value; 
Microsoft.Office.Interop.Word.Document doc = wordApp.Documents.Open(ref file, ref nullobj, ref nullobj, ref nullobj, ref nullobj, ref nullobj, ref nullobj, ref nullobj, ref nullobj, ref nullobj, ref nullobj, ref nullobj, ref nullobj, ref nullobj, ref nullobj, ref nullobj); doc.ActiveWindow.Selection.WholeStory(); doc.ActiveWindow.Selection.ClearFormatting(); doc.ActiveWindow.Selection.Copy(); DOTextData doc.ActiveWindow.Selection.Text.Trim() + " ";

doc.Close(ref nullobj, ref nullobj, ref nullobj); wordApp.Quit(ref nullobj, ref nullobj, ref nullobj);

\#endregion "Reading word data..."

Now the DOTextData will contain the word data as a string .This is used to update CLOB [7.3.2].

\subsection{Saving Data In Database}

To save data, two functions are used, one to insert and other to update [25][26]. The insert C\# function will call PLSQL procedure [3] to save basic details such as Daily Orders Part I no., type and date. This function will insert the BLOB, CLOB with EMPTY_BLOB(), and EMPTY_CLOB() function.

The update $\mathrm{C} \#$ function will select the particular record for update and write file bytes for BLOB, extracted word data to CLOB and headings to CLOB [25]. The C\# function erases each BLOB and CLOB first and writes the bytes as below.

\subsubsection{Updating $B L O B$}

blob $=$ reader.GetOracleBlob $(0)$;

blob.Erase();

blob.Write(cPARAM_PDF, 0, cPARAM_PDF.Length);

blob.Flush();

blob.Close();

blob.Dispose();

cPARAM_PDF Byte Array is passed by the calling function from "Fileupload" control by using fuPdf.FileBytes.

\subsubsection{Updating $C L O B$}

byte[] newvalue $=$

System.Text.Encoding.Unicode.GetBytes(DOTextData);

$\mathrm{clob}=$ reader.GetOracleClob $(0)$;

clob.Erase();

clob.Write(newvalue, 0, newvalue.Length);

clob.Flush();

reader.Close();

reader.Dispose();

\subsection{Search (AND and OR)}

Using the search interface AND, OR search can be performed.

string smatter $=$ txtHeadReq.Text.Trim () ;

string sAllReg $=$ txtAllReq.Text.Trim();

string sAnyReg $=$ txtAnyReq.Text.Trim();

string qry = null;

string[] saAllRequiste = sAllReg.Split(' ');

string[] saAnyReg = sAnyReg.Split(' ');

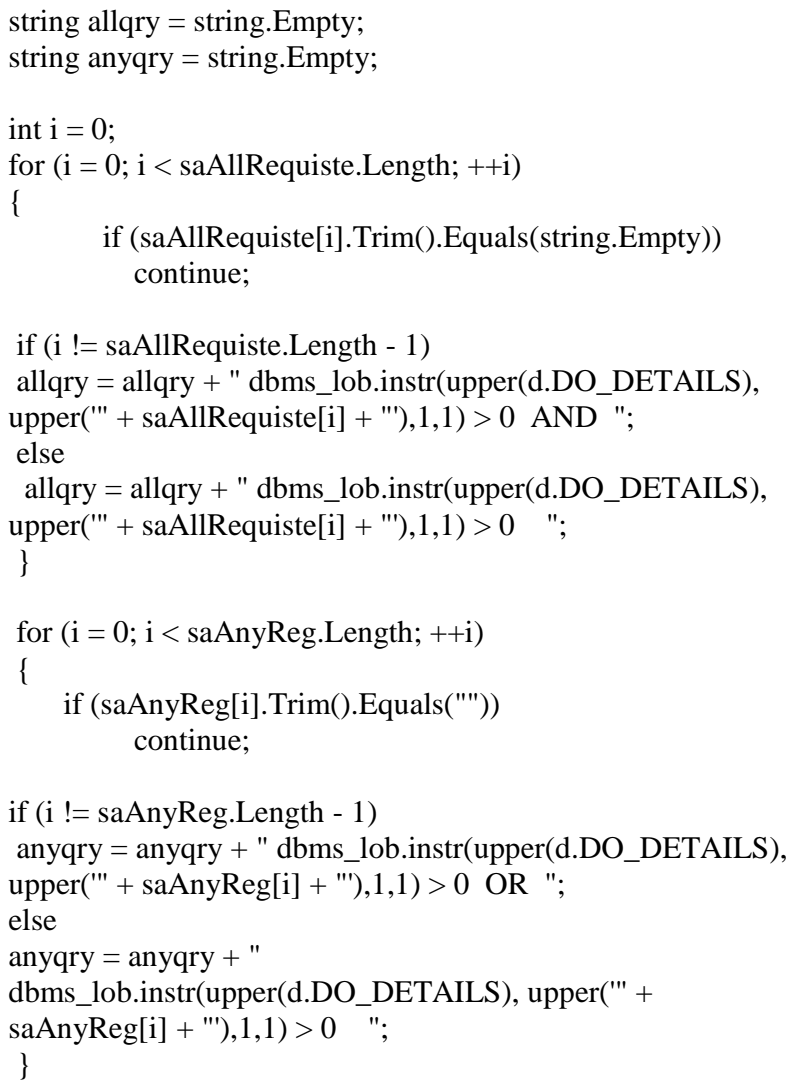




\subsection{Downling Requested File}

The file view module interface querying the database using oracle data adapter and load the result to a datatable.Code for A sending pdf file to client browser is as below.

DoPartDB db = new DoPartDB();

db.open();

$\mathrm{t}=\mathrm{db}$.getPDFFromDatabaseInt(cdate, dtype, do_number); db.close();

if $(\mathrm{t} !=$ null \&\& t.Rows.Count $>0)$

\{

string type $=$ t.Rows[0][1].ToString();

string do_type_rs $=$ t.Rows[0][2].ToString();

string FILENAME = do_number + "-" + do_type_rs + type;

Byte[] bytes $=($ Byte[])t.Rows[0][0];

context.Response $\cdot$ Buffer $=$ true;

context.Response. Charset = "utf8";

context.Response.Cache.SetCacheability(HttpCacheability.Pu blic);

context.Response.ContentType = "application/pdf";

context.Response.AddHeader("content-disposition",

"inline;filename=" + FILENAME);

context.Response.BinaryWrite(bytes);

context.Response.Flush();

context.Response.End();

\section{CONCLUSION AND FUTURE WORK}

This paper describes the methodologies, software, libraries and key codes to build a simple web based information system. It is an effective system and achieved the requirements [16].The system implemented have right alignment [15] with the existing non computerized system.

In next phase the system will be improved to handle the following.

It is noted that management and employees find difficulties in searching particular matter and availing the documents of fax, Inter Office Notifications and correspondence letters.

The existing fax automation systems in CVRDE manipulate all files in pdf format. An Interface system can be designed to manipulate pdf [4] and store this file in the new system with search words.

To implement the above features it is necessary to create different type of task holders and isolation of Information between task holders and common user. When the system handles these much document and data, the database becomes very big, proper achieving scheme and implementation is also studied and implemented.

\section{REFERENCES}

[1] Nick Randolph, David Gardner, Professional Visual Studio® 2008., Wiley Publishing Inc.

[2] Laurence Moroney ,Beginning Web Development, Silverlight, and ASP.NET AJAX From:Novice to Professional ., Apress Publishing.

[3] Steven Feuerstein , Bill Pribyl ,Oracle PL/SQL Programming Second Edition., By.O'Reilly \& Associates Publishing.
[4] Brno Lowagie, iText in Action CREATING AND MANIPULATING PDF., Manning Publications Co.

[5] Abraham Silberschts,Henry F.Korth,S.Sudarshan, "Databse System Concepts",fifth Editin,Pages 609-631

[6] Bear Bibeault and Yehuda Katz.," jQuery in Action”.Second edition 2008. Manning Publications Co.

[7] Suprotim Agarwal ,51 Tips, Tricks and Recipes with jQuery and ASP.NET Controls., A2Z Knowledge Visuals Pvt. Ltd.

[8] Batini Batini, Barone Daniele, Cabitza Federico and Grega Simone., Data Quality Methodology for Heterogeneous Data, International Journal of Database Management Systems ( IJDMS ), Vol.3, No.1, February 2011.

[9] Fraternali ,P.,Paolini,P.:Model-driven development of Web application:the AutoWeb system.ACM Transaction on Information System(TOIS)Volume 18,Issue 4, Pages $1-53(2000)$

[10] Legris, P.; Ingham, J.; Collerette, P.,

"Why do people use information technology? A critical review of the technology acceptance model "

Information \& Management, Volume 40, Issue 3 January 2003, Pages 191-204

[11] Jenkin, T.A.; Webster, J.; McShane, L.

"An agenda for'Green'information technology and systems research"

Information and Organization, November 2010

[12] Kamhawi, E.M.,

"The three tiers architecture of knowledge flow and management activities "

Information and Organization, Volume 20, Issue 3-4, July 2010, Pages 169-186

[13] Bimal Aklesh Kumar, "Thin Client Web-Based Campus Information Systems For FIJI National University "., International Journal of Software Engineering \& Applications (IJSEA), Vol.2, No.1, January 2011

[14] Badariah Solemon,Shamsul Sahibuddin,and Abdul Azim adb Ghanni, "Requirements Engineering Problems and Practices in Software Compnies:An Industry Survay".,International Conference on Advanced Software Engineering and Its Application,ASEA2009,Jeju Island ,Korea,December 2009.

[15] Lerina Aversano,Carmine Grasso, and Maria Tortorella "Framework for measuring the Aligment between Business Processes and Software System"., International Conference on Advanced Software Engineering and Its Application,ASEA2009,Jeju Island ,Korea,December 2009.

[16] Joseph Kibombo Balinkuddembe and Antoine Bagula:"Aligning the Software Project Selection Process with the Business Strategy: A Pilot Study"., International Conference on Advanced Software Engineering and Its Application,ASEA2009,Jeju Island ,Korea,December 2009.

[17] http://msdn.microsoft.com/en-us/library/bb398986.aspx. 
[18] http://msdn.microsoft.com/en-us/library/aa306178.

[19] http://msdn.microsoft.com/en-us/library/e1f13641.aspx.

[20] http://msdn.microsoft.com/en-us/library/8bs2ecf4.aspx (CLR)

[21] http://www.oracle.com/webfolder/technetwork/tutorials/ obe/db/dotnet/debugging/Debugging.htm .

[22] http://docs.jquery.com/Plugins/Calendar
[23] http://jqueryui.com/demos/dialog/\#modal

[24] http://www.artviper.net/website-tools/bumpboxlightbox.php

[25] http://www.codeproject.com/Articles/13675/Using-Cfor-Inserting-CLOB-Data-in-Oracle

[26] http://www.oracle.com/webfolder/technetwork/tutorials/ obe/db/hol08/dotnet/getstarted-c/getstarted_c_otn.htm 